\title{
An Evaluation of the Annual Performance of Higher Education Institutions Using the Data Envelopment Analysis "DEA" Method (Applied Study on Algerian Higher Education Institutions)
}

\author{
Imane Bebba \\ Institute of Economic and Commercial \\ sciences and Management science \\ Centre University Illizi-Algeria \\ imanbebba@yahoo.com
}

Ilyes Bensaci

Faculty of Economic and Commercial sciences and Management science

University Kasdi Merbah Ouargla-Algeria

ilyes12@gmail.com

\author{
Abdelghani Benhamed \\ Institute of Economic and Commercial \\ sciences and Management science \\ Centre University Illizi-Algeria \\ benhamed_2006@yahoo.fr
}

Abdelhak Bentafat

Faculty of Economic and Commercial sciences and Management science

University Kasdi Merbah Ouargla-Algeria

bentabdelhak@yahoo.fr

Received 29/5/2019

Accepted 26/6/2019

\begin{abstract}
:
This paper aims to clarify how to evaluate the Algerian institutions of higher education performance using a data envelopment analysis method based on the concept of benchmarking. To achieve this the study relied on the five inputs and outputs values of quantity, and reflect the dimensions of the teaching, learning and scientific research and are : total of students enrolled in graduation, and students enrolled in post graduation, permanent professors, students graduates, and scientific publications. After applying the data envelopment analysis method, the study reached models that there is variation in the performance of the Algerian institutions of higher education from one academic year to another, and its weak performance is caused by either inefficient internal processes or poor environmental conditions.
\end{abstract}

Keywords: Data Envelopment Analysis Method, Performance Indicators, Annual Performance Evaluation, Institutions of Higher Education, Algeria. 


\section{تقييم الأداء السنوي لمؤسسات التعليم العالي باستخدام أسلوب التحليل التطويقي للبيانات}

\section{(دراسة تطبيقية على مؤسسات التعليم العالي الجزائرية)}

\author{
عبد الغني بن حامد \\ العلوم الاقتصادية والتجارية وعلوم التسيير \\ المركز الجامعي إليزي - الجزائر \\ benhamed_2006@yahoo.fr
}

عبد الحق بن تفات

العلوم الاقتصادية والتجارية وعلوم التسبير

جامعة قاصدي مرباح ورقلة-الجزائر

bentabdelhak@yahoo.fr

قبول البحث

\author{
إيمان بية \\ العلوم الاقتصادية والتجارية وعلوم التسيير \\ المركز الجامعي إليزي - الجزائر \\ imanbebba@yahoo.com
}

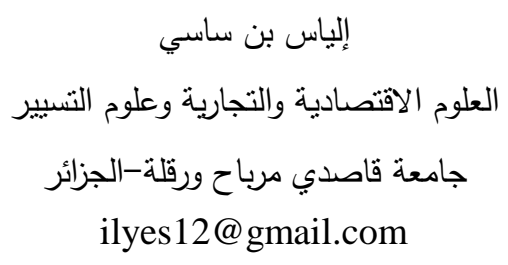

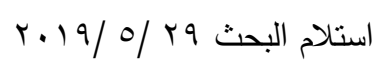

الملخص:

تهدف هذه الورقةُ البحثية إلى توضيح كيفية نقييم أداء مؤسسات التعليم العالي الجزائرية باستخدام أسلوب التحليل التطويقي للبيانات المستتد

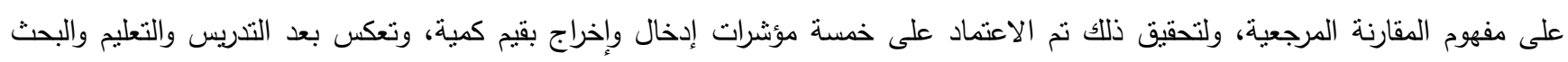

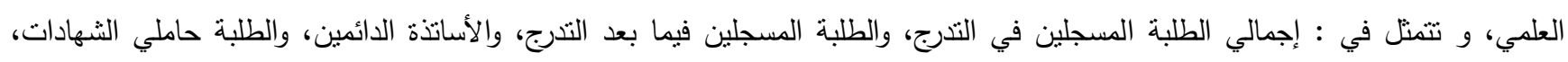
والمنشورات العلمية. وتم التوصل بعد تطبيق نماذج أسلوب التحليل التطويقي للبيانات إلى وجود نباين في أداء مؤسسات التعليم العالي الجزائرية

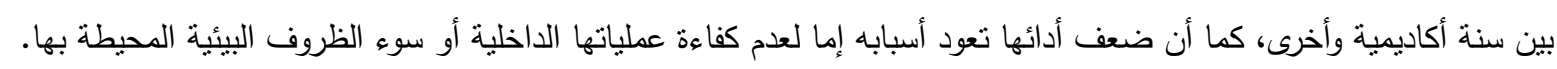

الكلمات الدفتاحية: أسلوب تحليل تطويقي للبيانات، مؤشرات أداء، تقييم أداء سنوي، مؤسسات تعليم عالي، جزائر .

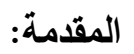

نسبة المخرجات إلى المدخلات، شرط أن يتم قياس جميع المدخلات والمخرجات بشكل صحيح" (2). يتبين من خلال تعريف Farrell( (ب) أنَّ المؤسسة ذات الكفاءة العالية هي التي نجحت في إنتاج أكبر قدر من الدخرجات من خلد استخدامها لكمية معينة من المدخلات.

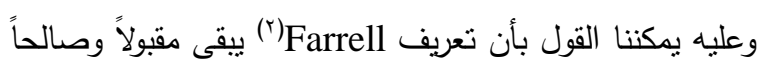
للنطبيق إذا نوفرت في المؤسسة أو النظام مخرجات متعددة متجانسة، ومدخلات متعددة متجانسة وبأوزان نسبية معروفة.

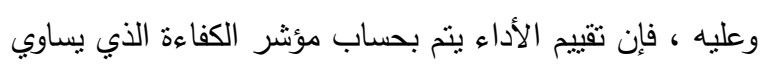

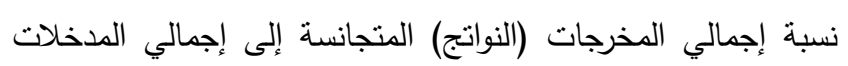
المتجانسة (3).
لقد ارتبطت النظرة التقليدية لمفهوم الأداء بالجانب المالي،

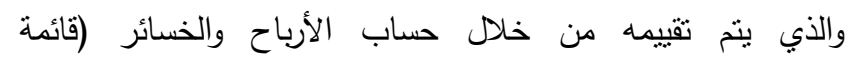

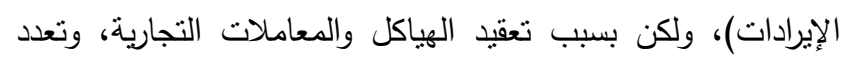

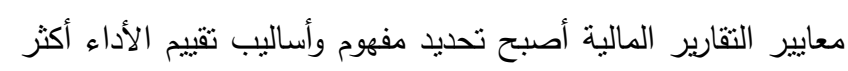

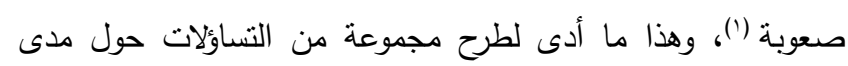

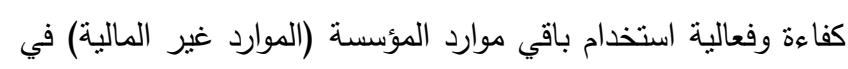
عملية تقييم الأداء. ومن هنا بدأ التفكير في نهج وأساليب جديدة لتقيبم وقياس أداء

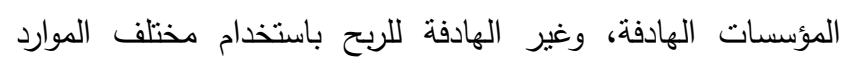

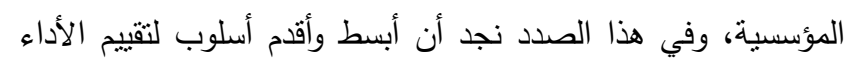

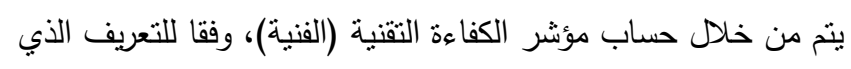
قدمه Farrell في سنة 90 و والذي ينص على على أن : "الكفاءة تمنل 
الاحصائية القياسية لم يتحصل على نتائج مرضية لتقييم كفاءة هذا

$$
\text { البرنامج في كل مدرسة(؛). }
$$

ومن هنا بدأ في التفكير عن أسلوب أكثر نجاعة، حيث أعاد

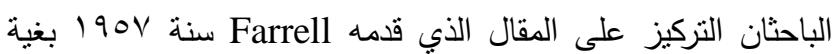
نطوير أفضل أساليب ونماذج نقييم الانتاجية، كما قام الباحثان بإعادة النظر والتدقيق في عمل سابق جمع المشرف على الأطروحة Charnes و هذا العمل يقدم معيارا يعطي شكلا حسابيا

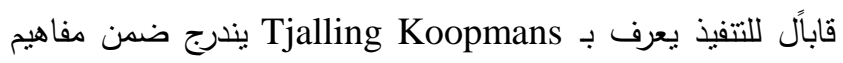

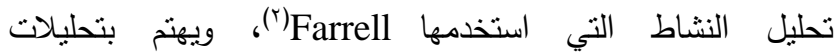
الاقتصاديات بأكملها.

ومع تضافر جهود الباحثين الثلاثة تم التوصل إلى نتيجة

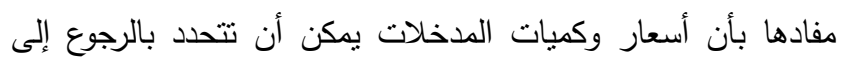

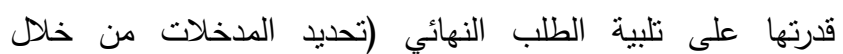
المخرجات) بل الأهم من ذلك يمكن استخدام أداء وحدات اتخاذ القرار

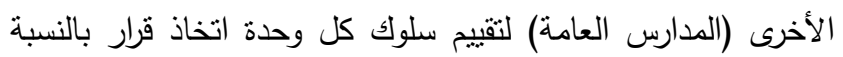

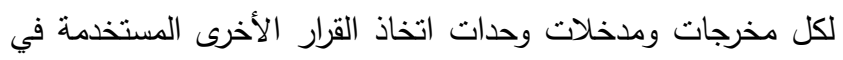
الدراسة. وهذا ما بمكنهم من تحديد كفاءتها النسبية تجريبيا(؛). Charnes, وفي سنة 1971 نشر مقال علمي للباحثين European Journal of بمجلة Cooper, Rhodes Operations Research للبيانات (The Data Envelopment Analysis) لأول مرة، والذي فلي لئي

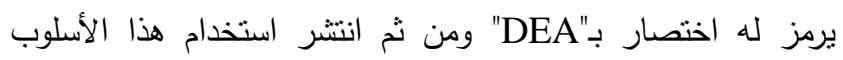

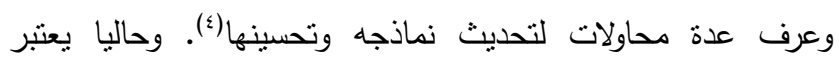
أسلوب الـ DEA أحد الأساليب الأكثر استخداما في تحليل كفاءة

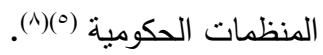
ومن خلال الاطلاع على بعض الدراسات السابقة تم ملاحظة

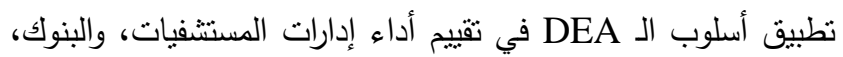

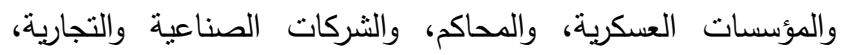

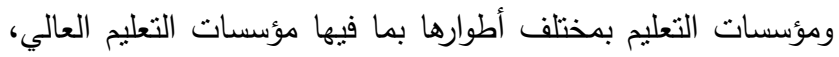
إضافة إلى استخدامه في تقييم اقتصاديات البلدان فيما بينها والأقاليم الجغرافية...الخ.

\section{1. مفهوم أسلوب التحليل التطويقي للبيانات.}

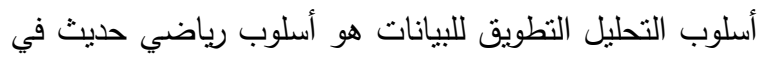
ميدان نماذج الإدارة الكمية(؟). وهو أحد تقنيات البرمجة الخطية، كما ينظر إليه كنهج يستند على البيانات الموجهة "data-oriented" لنقييم أداء مجموعة من التهات

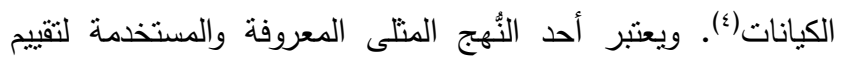
الكفاءة النسبية لمجموعة من وحدات اتخاذ القرار المتماتلة، والمقارنة
ويمكن ترجمة التعريف السابق في المعادلة التالية:

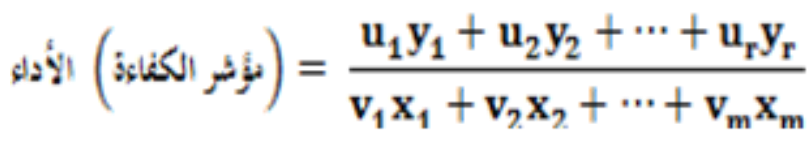

حيث إن : y هي المخرجات؛ و x هي الدخلات؛ و ( u (u هي $_{2}, \ldots u_{r}$

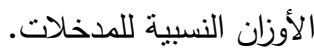

ورغم وضوح ودقة المعادلة أعلاه إلاّ أن عملية تقييم أداء

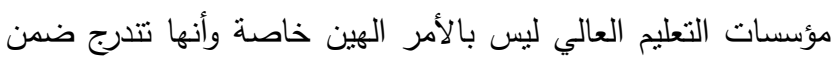
المنظمات المعقدة والتي نستخدم مدخلات متعددة ومنباينة لإنتاج

مخرجات متعددة ومنباينة(").

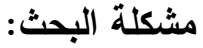

من خلال ما نم الإثارة له يمكن صياغة مشكلة الدراسة في

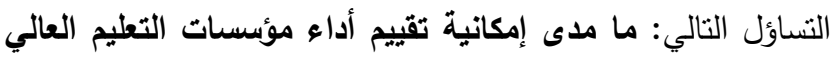

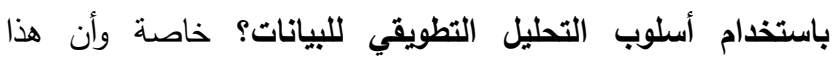

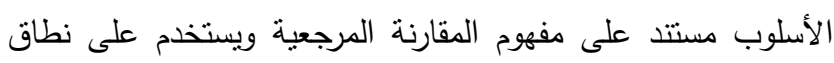
واسع في تقييم أداء العديد من المؤسسات الهادفة وغير الهادفة للربح. وبغية الإجابة على مشكلة الدراسة تم التطرق في الجانب

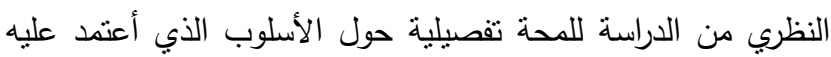

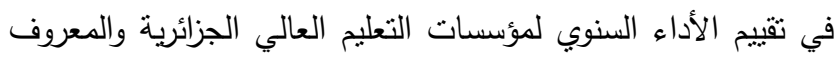
بأسلوب التحليل التطويقي للبيانات وكيفية استخدامه في تقييم أداء المؤسسات بصفة عامة، أما الجانب الثاني فقد نم تخصيصه للإناسنة التطبيقية والتي استُخدم فيها أسلوب التحليل التطويقي للييانات لنقييم أداء مؤسسات التعليم العالي الجزائرية خلال 17 سنة أكاديمية متتالية. أولاً: أدوات وطريقة الدراسة (لمحة تفصيلية حول أسلوب

\section{التحليل التطويقي للبيانات).}

أسلوب التحليل النطويقي للبيانات، هو نتاج لجهود أطروحة دكتوراه معدة من طرف Rhodes Edwardo وتحت إثراف Carnegie Mellon University's بجامعة Cooper William School of Urban \& Public Affairs موجهة لتقييم البرامج التعليمية التي تقدم للطلبة الدحرومين والأقل

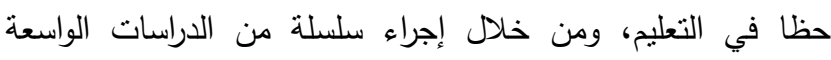
النطاق على عينة من المدارس العامة في الولايات المتحدة المتمانلة،

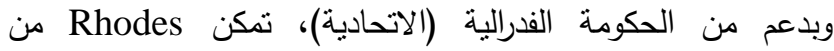
الوصول إلى أكبر قاعدة بيانات كمية تضم متغيرات إدخال وإخراج

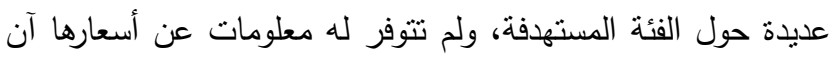
ذالك؛ ولهذا السبب واجه الباحث صعوبة كبيرة في قياس الكفاءة تطبيقيا، وحتى بعد عدة محاولات واستخدامه للهموعة من النُّجج 
هناك أي زيادة في المدخلات، سينتج عنها زيادة متتاسبة في المخرجات(9).

\section{Banker Charnes Cooper ) BCC r., نموذج الـ r.}

.(Model

نظرا لانتشار استخدام أسلوب التحليل التطويقي للبيانات،

وتواصل الأبحاث حوله قام كل من \&anker, Charnes \& Cooper

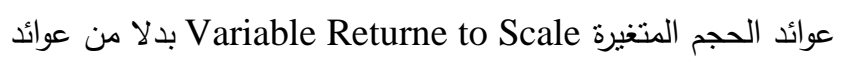
الحجم الثابتة، لأنه من غير المنطقي أن تعمل كل المؤسسات عند الداند

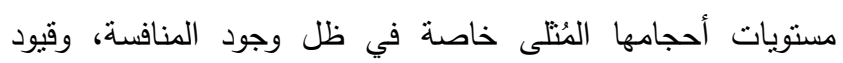
مفروضة سواء كانت حكومية، أو مالية أو غيرها من القيود الأخرى.

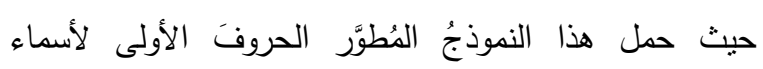

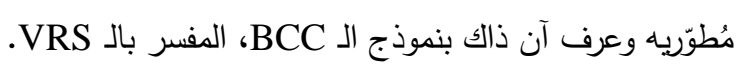

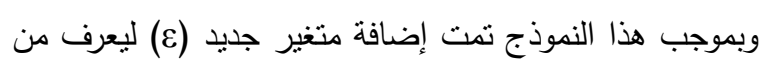

خلاله عوائد الغلة المتغيرة لوحدة اتخاذ القرار قيد الدراسة (10). كما يميز هذا النموذج بين نوعين من الكفاءة هما: الكفاءة الفنية

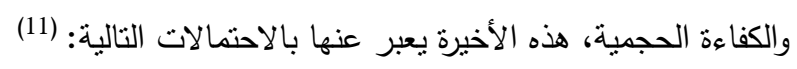

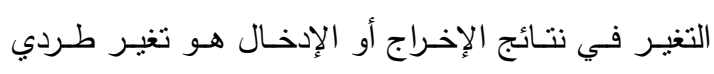

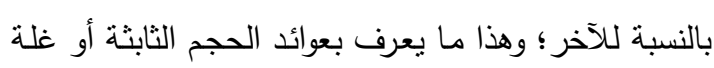

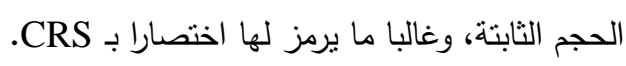

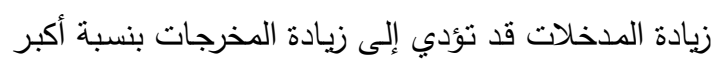

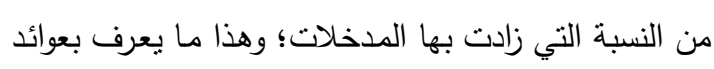

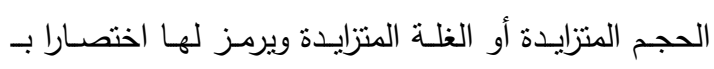

IRS

زيادة الدخخلات قد تؤدي إلى الزيادة في المخرجات بنسب منخفضة؛ وهذا ما يعرف بعوائد الحجم المتتاقصـة أو غلة فئل

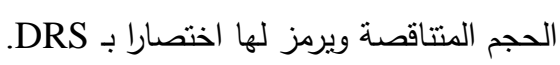
ويمكن تطبيق نموذجي الـ CCR، والـ BCC وفقا لنوعية توجيه

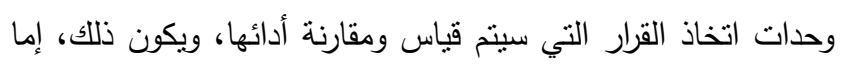

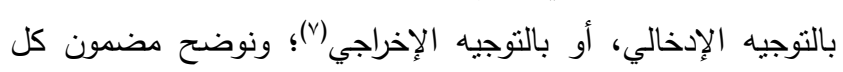

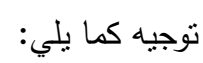

التوجيه الاخخالي (Input-Oriented): يقصد به بيه فياس

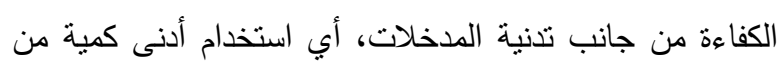
المدخلات لتحقيق كمية معينة من الخدمات (أو المخرجات).

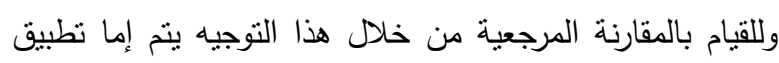
نموذج يعرف بـ (CCR-I) والذي يفترض عوائد الحجم الثابثة

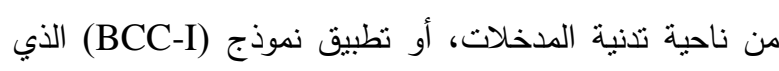
يفترض عوائد الحجم المتغيرة من ناحية تدنية المدخلات.

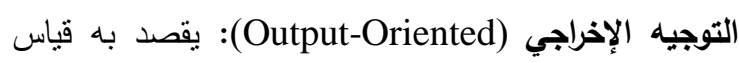
الكفاءة من جانب تعظيم المخرجات، أي الكمية التي يمكن من بل بله

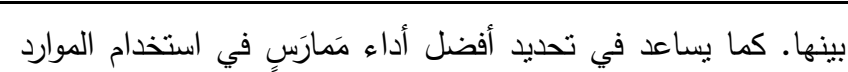
بين مجموعة منماثلة من المنظمات أو وحدات اتخاذ القرار .

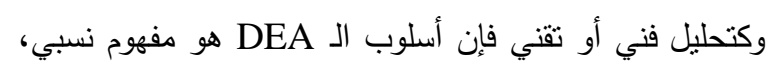

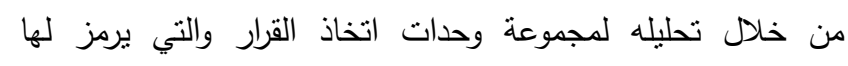

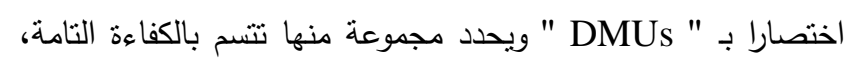
وتعتبر هذه الأخيرة وحداتٍ مرجعيةً لباقي الوحدات غير الكفؤة. أما

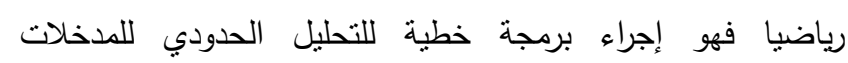

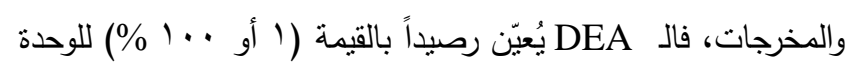

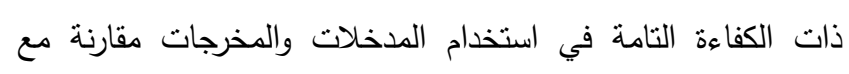
الوحدات الأخرى، ويعين رصيداً يختلف عن القيمة واحد ( ) للوحدات التهات غير الكفؤة (6).

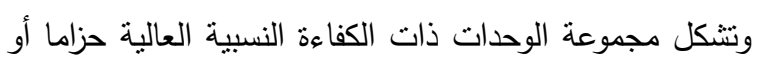

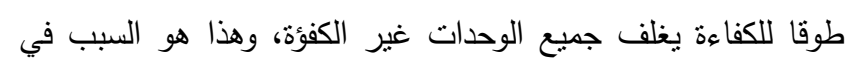

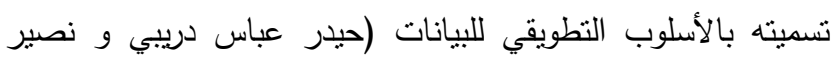

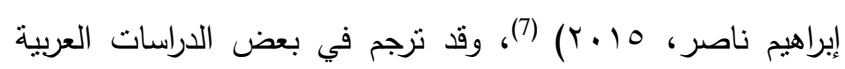
السابقة التي اطلعنا عليها تحت اسم تحليل مغلف البيانات.

\section{r أ. النماذج الأساسية لأسلوب التحليل التطويقي للبيانات.}

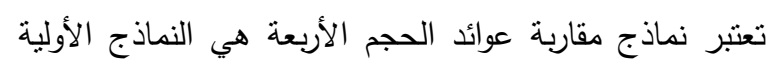

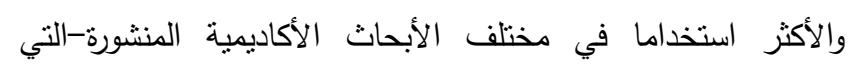

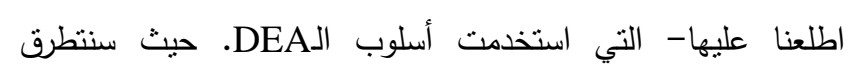
لمضمون كل نموذج باختصار كما يلي:

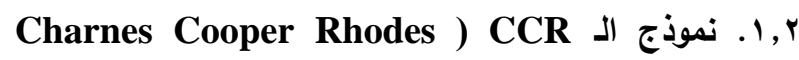

\section{.(Model}

يعد نموذج الـ CCR أول نموذج طبق وفقه أسلوب الـDEA،

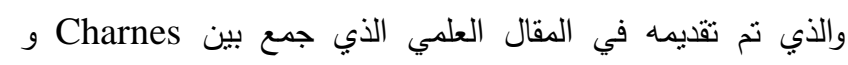
النة Rhodes و Cooper استخدم هذا النموذج آن ذاك لتقييم برنامج يعرف بـ" Program Follow Through

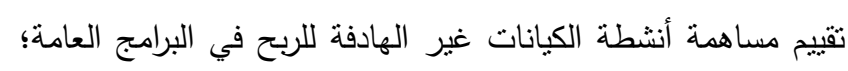

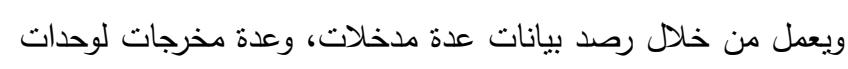

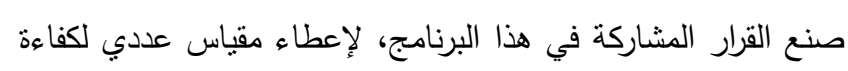

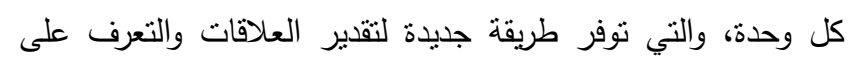
أوجه القصور (8). هذا النموذج الذي يرمز له بـ CCR اختصار لأسماء الباحثين

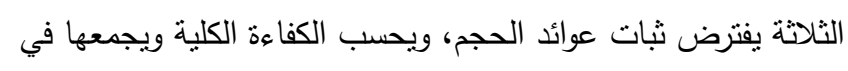

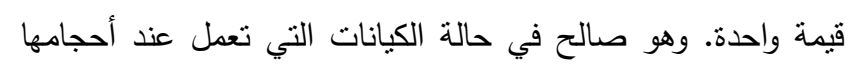

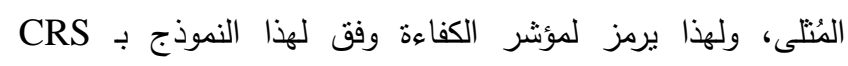

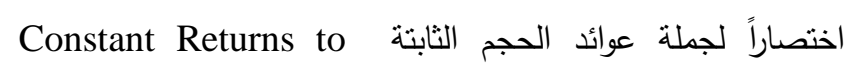

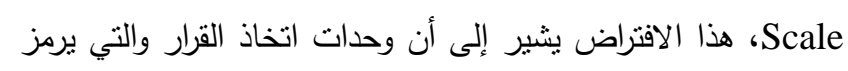
لها اختصارا بـ "DMU" تعمل تحت عوائد حجم ثابتة أي إذا كانت الترات 
منهجية موزعة على الحدود بدلا من النزعة المركزية؛

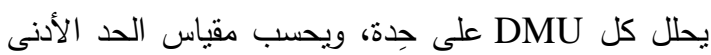
أو الأقصى من أداء كل وحدة؛

بديل رئيسي يغني عن استخدام حدود التكلفة العشوائية، لأنه أسلوب غير حدودي، ويتميز على نهج الحدود

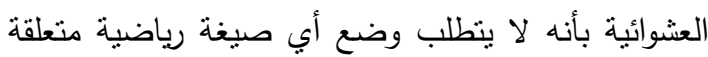

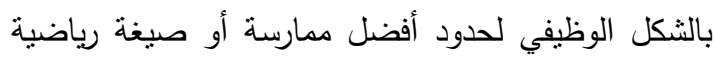
للادلة الني تربط بين متغيرات الإدخال ومتغيرات الإخراج. Cooper \& Seiford \& Zhu, إضافة إلى المزايا الني قدمهات لإنيات

$$
\text { : (r.11) }
$$

تعريف الـ DMU يتميز بخاصيتي الثمول، والمرونة؛

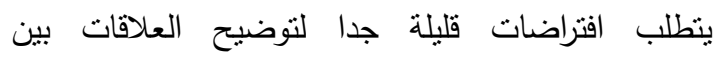
المدخلات المتعددة والمخرجات المتعددة المرتبطة بوحدات

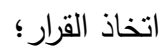
تعريف الكفاءة النسبية وفقا لأسلوب DEA يجنب الحاجة

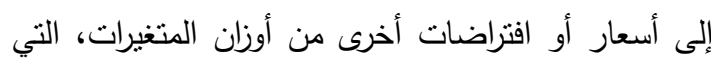

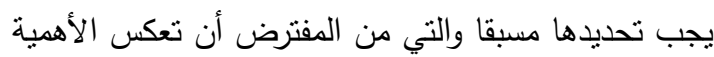
النسبية لدختلف الدخخلات والمخرجات؛ يجنب الحاجة لتحديد وتوضيح العلاقات الرسمية الني يفترض أن توجد بين المدخلات والمخرجات.

كما يضيف حيدر عباس(") أن أسلوب الـ DEA له مزايا تتمنل

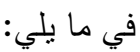

يجمع في قياسه للكفاءة بين الكفاءة الداخلية بشقيها (الكمية والنوعية)، وبين الكفاءة الخارجية؛ حيث بمكن للأسلوب

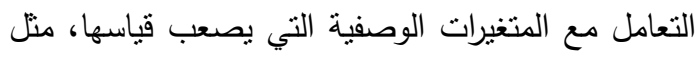

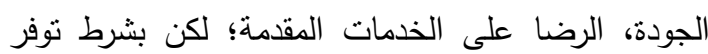

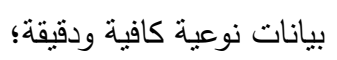
يمكنه التعامل مع العوامل الخارجة عن تحكم الوحدة المراد

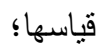
يحدد مصادر وكمية الطاقة الراكدة من المدخلات المستخدمة من قبل الوحدات الأقل كفاءة؛ يحدد مصادر وكمية الطاقة الفائضة أو إمكانية زيادة المخرجات في الوحدات الأقل كفاءة وبدون زيادة

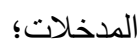
يحدد طبيعة العائد على حجم الإنتاج عند حدود الكفاءة (عائد ثابت أو متغير ). •. حدود استخدام أسلوب الـ DEA.

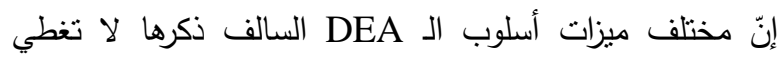
بعضا من أوجه قصوره، والمتمنلة فيما يلي:
خلالها الزيادة في كمية المخرجات المحققة دون الحاجة للتننية من كمية الدذخلات المستخدمة، أو بعبارة أخرى قياس كفاءة

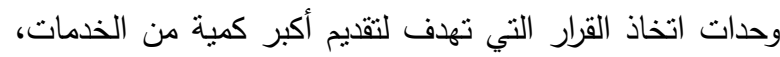

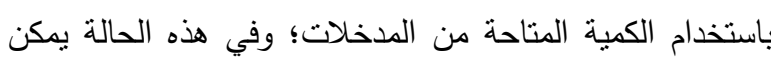

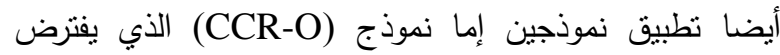

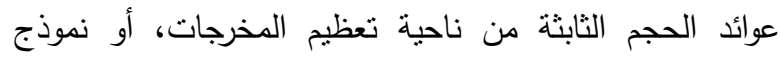
الذي يفترض عوائد الحجم المتغيرة من ناحية تعظيم

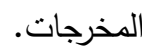

r. الفرق بين نماذج مقارية عوائد الحجم. أول فرق يمكن استتتاجه من خلال مفهوم كل نموذج، يتمثل

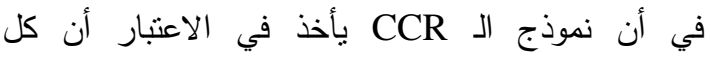

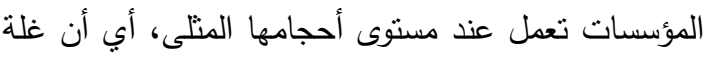

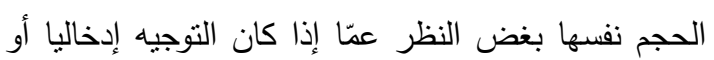

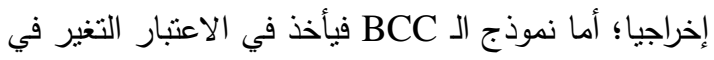
غلة الحجم، فقد تكون مثتاقصة، أو ثابتة، أو مثزايدة.

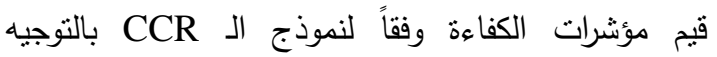
الإدخالي، وبالتوجيه الإخراجي تكون نفسها على الدوام. وعليه فإن تطبيق أحد التوجيهين يفي بالغرض، لكن لإنى كثيرا ما تختلف التقييمات باختلاف نوع التوجيه (إدخالي أو لوان

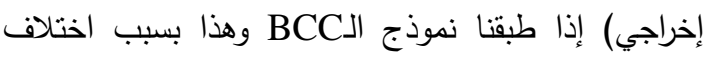

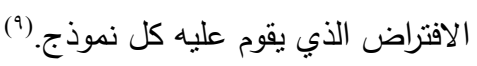

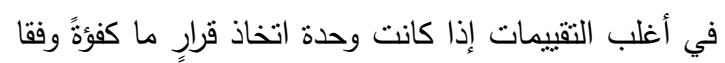

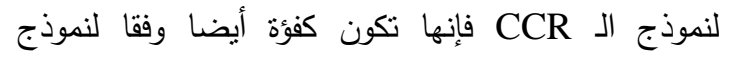

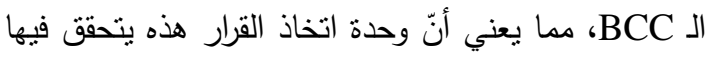

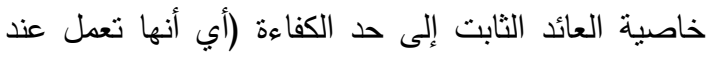

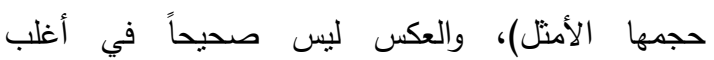

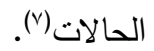
مقياس الكفاءة الناتج عن نموذج الـ BCC يعبر عن الكفاءة

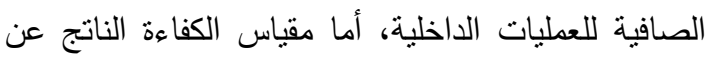

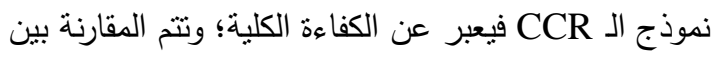
النموذجين لمعرفة مصادر عدم كفاءة الوحدات غير الكفؤة: هل هو راجع إلى عدم كفاءة العمليات الداخلية لهذه

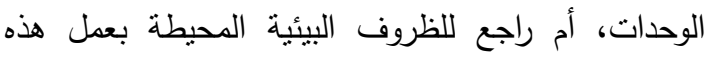

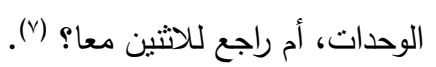

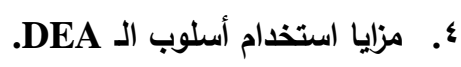

من خلال مفهوم أسلوب الـ DEA السالف عرضه يمكننا القول

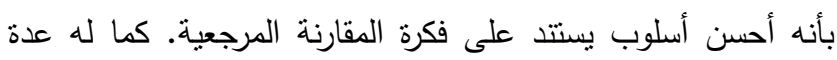

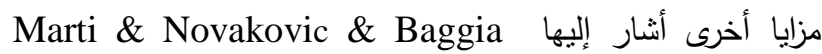

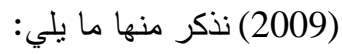


فترة زمنية وكيانات متعددة.

أما فيما يخص القواعد الأساسية لضمان نجاح نطبيق نماذج أسلوب الـ DEA فتتمثل في ثلاثة قواعد (') فيما يلي : القاعدة الأولى : يجب أن يكون عدد وحدات اتخاذ القرار الداخلة في الدراسة أكبر من أو يساوي حاصل جداء الدخلات

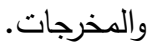

$$
\text { S }
$$

القاعدة الثانية : يجب أن يكون عدد وحدات اتخاذ القرار الداخلة في الدراسة) أكبر من أو يساوي ضعف مجموع المدخلات

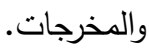

$$
\mathrm{S}_{\mathrm{s}} \geq 2(\mathrm{I}+\mathrm{O}) \quad \text { أي أن }
$$

القاعدة الثالثة : يجب أن يكون عدد وحدات اتخاذ القرار ذات

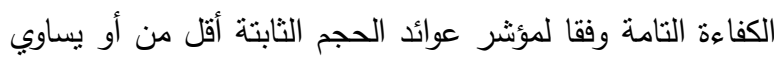

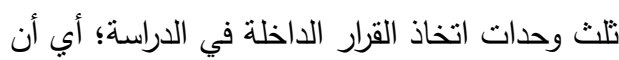
Eff DMUs $\leq 1 / 3 * \mathrm{~S}_{\mathrm{s}}$

$$
\begin{aligned}
& \text { O } \\
& \text { I } \\
& \text { : S } \text { : }
\end{aligned}
$$$$
\text { حيث إن }
$$

: Eff DMUs

من بين مختلف البرامج الحاسوبية المصممة خصيصا لقياس أداء مجموعة من وحدات اتخاذ القرار المتماثلة باستخدام أسلوب الـ "DEAP Version 2.1" تم الاستعانة بالبرنامج الحاسوبي DEA لتحقيق ذلك.

ثانيا: الدراسة التطبيقية تحليل ومناقثة نتائج قياس أداء مؤسسات التعليم العالي الجزائرية خلال 17 سنافئه أكاديمية باستخدام الـ DEA. بغية ربط الإطار النظري المعروض في الثق الأول من الورقة البحثية، وكذا إعطاء الدراسة طابعاً نطبيقياً يثبت أو ينفي مدى إمكانية

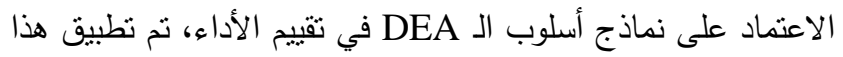
الأسلوب لنقييم أداء إجمالي مؤسسات التعليم العالي الجزائرية في كل سنة أكاديمية. ولتحقيق ذلك تم المرور بالمراحل التالية: 1. تديد مؤثرات (متغيرات) الإذخال والإخراج:

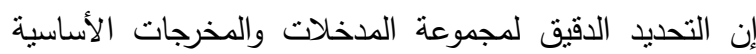
لنطبيق أسلوب التحليل النطويقي للبيانات يعطي نتائج نقييم أداء

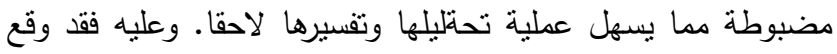

لعل أول صعوبة نواجه الباحث لدى رغبته في استخدام هذا

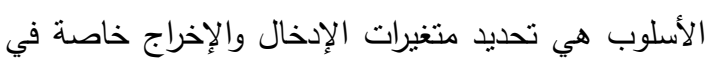
قطاع التعليم العالي الذي يضم متغيرات متعدد ومتداخلة الإنيات

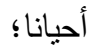

النماذج الأساسية لأسلوب النحليل النطويقي للبيانات (أي نموذج اللCCR بكلا التوجيهين، ونموذج الـ BCC بكلا التوجيهين) تقوم بتقييم الكفاءة النسبية لوحدات اتخاذ القرار

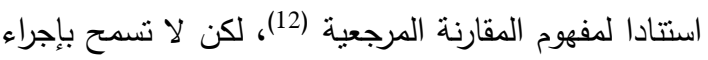

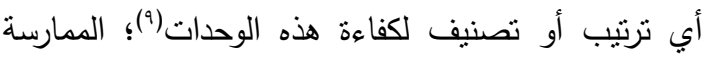
الثائعة لأسلوب الـ DEA هي باستخدام المدخلات التي يمكن للمسؤولين في المستويات العليا التحكم فيها فقط، التهاب

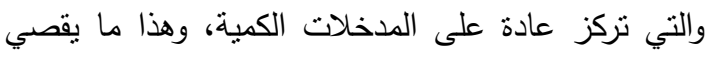
استخدام بيانات حول المدخلات، والمخرجات غير المادية، متل : الخبرات، والكفاءات، الجودة ...الخ في عملية تحليل وتقييم الكفاءة بالرغم من إمكانية استخدامها إذا ما توفرت

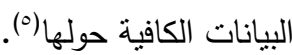

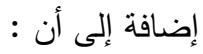

أسلوب الـ DEA ، يكثف عن هدى كفاءة الددخلات إلات المستخدمة لتحقيق المخرجات المطلوبة، لكن لا يكثف لنا

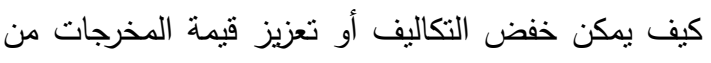
خلال اختبار توليفات مختلفة من المدخلات، والمخرجات المستخدمة(7).

ד. الثروط والقواعد الأساسية لقياس ومقارنة الأداء باستخذام

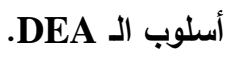

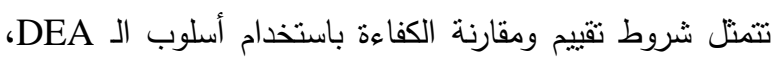
انطلاقا مما تم استتتاجه من مفهومه فيما يلي : توفر مجموعة من وحدات اتخاذ القرار المتماثلة والمتجانسة من حيث المدخلات، والمخرجات ولها نفس الهدف (نفس

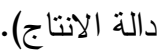
للحصول على الكفاءة في شكل أرقام (معاملات، أو نسب) يجب أن نكون الدخلات، والمخرجات المطبق عليها أسلوب الـ DEA عبارة عن قيم موجبة قابلة للقياس الكمي. توفر علاقة خطية وطردية بين الدخلات والدخرجات، بحيث أن كل زيادة في المدخلات سنساهم في زئة زيادة

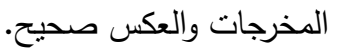
أما Rosenmayer Tomáš (2014) فيرى أنه يمكن تقييم ومقارنة الكفاءة النسبية في إحدى الحالات التالية: فترة زمنية لنفس الكيان؛ كيانات متعددة في نفس السنة؛ 
المنشورات العلمية: ويشير لإجمالي الإنتاجية العلمية السنوية والوثائق والبحوث العلمية، والأوراق العلمية المقدمة في الملتقيات

$$
\begin{aligned}
& \text { المنشورة والمتوفرة على مواقع الويب. } \\
& \text { r. تحديد وحدات اتخاذ القرار: }
\end{aligned}
$$

بالنسبة لوحدات اتخاذ القرار والتي تعكس عينة الدراسة التي

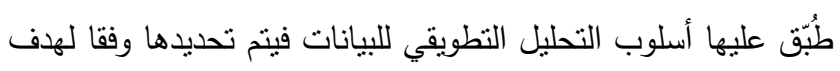
الدراسة، فقد تكون مجموعة من الكيانات المتثابهة خلال سنة واحدة وقد تكون عدة سنوات تعكس متغيرات كيان واحد، وقد تكون عدة كيانات تعكس فترة زمنية، تشمل جميع المذكرات والأطروحات والمقالات .وفي الدراسة الحالية فقد كانت وحدات اتخاذ القرار عبارة عن 17 سنة أكاديمية تضم مؤشرات إدخال وإخراج كافة مؤسسات

$$
\text { التعليم العالي الجزائرية. }
$$

\section{r. الخيص البيانات المجمعة:}

بعد تجميع مؤشرات الإدخال والإخراج القابلة للقياس الكمي، تم

$$
\text { تلخيصها في الجدول رقم (1). }
$$

الاختيار في هذه الدراسة على ثلاث مدخلات وأربعة مخرجات،

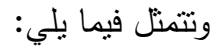

1,1. مؤشرات (متغيرات) الإدخال : تم اختيار ثناثة مدخلات تعتبر مرتكزات أساسية في أي مؤسسة تعليمية، وتعكس بُعْدَ عملية التدريس والتعلم، وهي :

الطلبة المسجلون في مرحلة التدرج : بشمل العدد الإجمالي للطلبة المسجلين في مرحلة الليسانس. الطلبة المسجلون في مرحلة ما بعد التدرج : يضم كافة المسجلين في برامج الماجستير ، والدكتوراه. الأساتذة الدائمون (أو الموظقون الأكاديميون) : أب العدد الإجمالي لأعضاء هيئة التدريس بدوام كامل، بمختلف الرتب الأكاديمية.

I, I. مؤشرات (متغيرات) الإخراج : وقع الاختيار على مخرجين مهمين ويعكسان بُعْد العمليات الأكاديمية والبحث العلمي، وهما:

\begin{tabular}{|c|c|c|c|c|c|c|}
\hline \multicolumn{2}{|c|}{$\begin{array}{c}\text { مؤشرات/متغيرات الإخراج } \\
\text { Outputs }\end{array}$} & \multicolumn{3}{|c|}{ مؤشرات/متغيرات الإخخال $\begin{array}{c}\text { Inputs } \\
\text { Ints }\end{array}$} & \multirow{2}{*}{\multicolumn{2}{|c|}{$\begin{array}{l}\text { وحدة اتخاذ القراد } \\
\text { DMU }\end{array}$}} \\
\hline إجمالي المنشورات العلمية & اجمالي حاملي الثهادات & إجمالي الأساتذة & $\begin{array}{c}\text { إجمالي المسجلين في مرحلة ما } \\
\text { التّرج } \\
\text { Input }_{2}\end{array}$ & $\begin{array}{c}\text { إجمالي المسجلين في } \\
\text { مرحلة التدرج } \\
\text { Input }_{1}\end{array}$ & & \\
\hline 518 & 52804 & 17460 & 20846 & 407995 & $\mathrm{DMU}_{1}$ & r... \\
\hline 593 & 65192 & 17780 & 22533 & 466084 & $\mathrm{DMU}_{2}$ & $r \ldots l$ \\
\hline 642 & 72737 & 19275 & 26060 & 543869 & $\mathrm{DMU}_{3}$ & $r \ldots r$ \\
\hline 883 & 77972 & 20769 & 26279 & 589993 & $\mathrm{DMU}_{4}$ & $r \ldots r$ \\
\hline 1162 & 91828 & 22650 & 30221 & 622980 & $\mathrm{DMU}_{5}$ & $r \ldots \varepsilon$ \\
\hline 1299 & 107515 & 25229 & 33630 & 721833 & $\mathrm{DMU}_{6}$ & r...o \\
\hline 1811 & 112932 & 27067 & 37787 & 743054 & $\mathrm{DMU}_{7}$ & $r \ldots r$ \\
\hline 2011 & 121905 & 29062 & 43458 & 820664 & $\mathrm{DMU}_{8}$ & $r \ldots v$ \\
\hline 2471 & 146889 & 31703 & 48764 & 952067 & $\mathrm{DMU}_{9}$ & $r \ldots \lambda$ \\
\hline 3108 & 150014 & 34470 & 54924 & 1048899 & $\mathrm{DMU}_{10}$ & $r \ldots q$ \\
\hline 3163 & 199767 & 37688 & 58975 & 1034313 & $\mathrm{DMU}_{11}$ & $r \cdot 1$. \\
\hline 3583 & 246743 & 40140 & 60617 & 1077945 & $\mathrm{DMU}_{12}$ & $r .11$ \\
\hline 4276 & 233879 & 44448 & 64212 & 1090592 & $\mathrm{DMU}_{1 »}$ & $r \cdot l r$ \\
\hline 4943 & 288602 & 48398 & 67671 & 1124434 & $\mathrm{DMU}_{14}$ & $r .1 r$ \\
\hline 5160 & 271430 & 51299 & 70734 & 1119515 & $\mathrm{DMU}_{15}$ & $r \cdot 1 \leq$ \\
\hline 5171 & 283430 & 53622 & 76510 & 1165040 & $\mathrm{DMU}_{16}$ & $r .10$ \\
\hline
\end{tabular}
حاملو الشهادات (المتخرجون): إجمالي تعداد الطلبة المتخرجين في مرحلة التدرج.

الجدول رقم ( 1 •): المؤشرات الكمية المجمعة لإجمالي مؤسسات التعليم العالي الجزائرية خلال 14 سنة الأكاديمية.

$16=S_{s}$

المصدر :

DDP/SDPP, ANNUAIRE STATISTIQUE, N (39-40-41-42-43-44), MESRS, REPUBLIQUE ALGERIENNE DEMOCRATIQUE ET POPULAIRE, (2009-2015) , \& http://www.scimagojr.com/countrysearch.php?country=dz (Date Found : 17/10/2017) 
التأكد من مدى صحة اختيار هذا الأسلوب، ومدى توفر شروط القوة التقبيرية للأسلوب، وعليه تم القيام بالخطوات النالية: §, 1. التأكد من توفر العلاقة الطردية بين متغيرات الإدخال

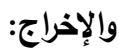

من أجل التأكد من وجود علاقة طردية بين متغيرات الدراسة، وجب التأكد من مدى وجود ارتباط بين متغيرات الإدخال والإخراج

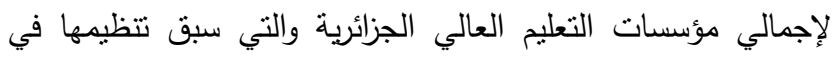

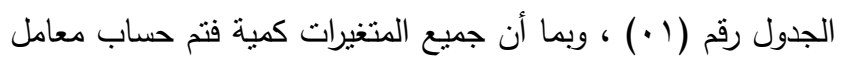
الارتباط بيرسون (r)؛؛ والنتائج المتحصل عليها نم توضيحها في النيات

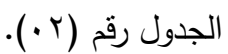

؛. التأكد من صحة استخدام أسلوب الـDEA في تقييم أداء مؤسسات التعليم العالي الجزائرية:

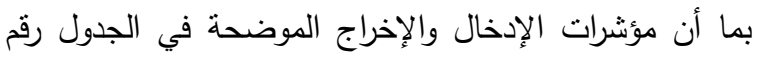

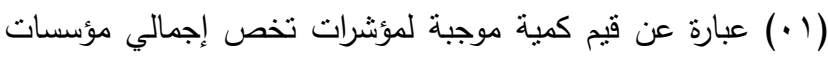

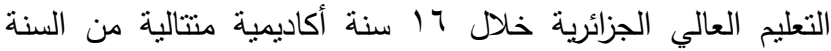

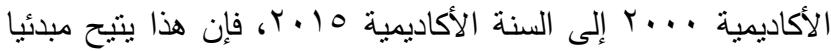
اختيار أسلوب الـ DEA لتقييم ومقارنة أداء هذه المؤسسات في كل

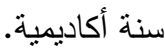
لكن قبل إدراج بيانات الجدول رقم ( ل • ) في البرنامج الحاسوبي

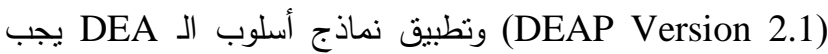

الجدول رقم (ץ •) : مصفوفة الارتباط بين متغيرات إدخال وإخراج مؤسسات التطليم العالي الجزائرية خلال 14 سنة أكاديمية.

\begin{tabular}{|c|c|c|c|c|c|}
\hline إجمالي المنشورات & حاملي الثهادات الطلبة & إجمالي الأساتذة & إجمالي الطلبة المسجلين & المسجلين في التّالي الطلبة & المتغيرات \\
\hline 0.9 १९** & $0.9 r \Gamma * *$ & $0.9 \leqslant 7 * *$ & $0.980^{* *}$ & 1.000 & إجمالي الطلبة المسجلين في التدرج \\
\hline $0.9 \wedge \vee * *$ & $0.9 \vee Y * *$ & $0.9 \wedge \wedge * *$ & 1.000 & $0.9 \wedge \cdot * *$ & إجمالي الطلبة المسجلين في ما بعد التدرج \\
\hline $0.997 * *$ & $0.9 \wedge \varepsilon * *$ & 1.000 & $0.9 \wedge \wedge * *$ & $0.9 \leq 7 * *$ & إجمالي الأساتذة الدائمين \\
\hline $0.9 \wedge \Upsilon * * *$ & 1.000 & $0.9 \wedge \leqslant * *$ & $0.9 \vee \Upsilon * * *$ & $0.94 r * * *$ & إجمالي الطلبة حاملي الشهادات \\
\hline 1.000 & $0.9 \wedge r * * *$ & $0.997 * *$ & $0.9 \wedge \vee * *$ & 0.9 १९** & إجمالي المنشورات العلمية \\
\hline
\end{tabular}

** La corrélation est significative au niveau 0.01

$$
\text { وفقا للقاعد الثانية يجب أن يكون : }
$$

$$
\begin{aligned}
& {\left[\mathrm{S}_{\mathrm{s}} \geq 2(\mathrm{I}+\mathrm{O})\right] \longrightarrow[16>2(3+2)] \longrightarrow[16>10]}
\end{aligned}
$$

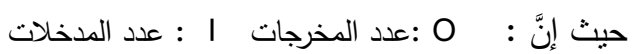

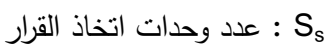

القاعدة الثانية محققة أيضا، لأن عدد وحدات اتخاذ القرار أكبر

$$
\text { من ضعف مجموع الدذخلات والمخرجات. }
$$

من خلال مختلف الخطوات السابقة اتضح أن الثروط الأساسية لاستخدام أسلوب الـ DEA ، وقواعد قوة التقدير لأسلوب الـ

$$
\text { DEA كلها منوفرة، مما يعني أنه: }
$$

هناك مؤشرات إدخال وإخراج تغطي 17 سنة أكاديمية (فترة زمنية) لكيان واحد مما يعني إمكانية قياس الأداء وإمكانية

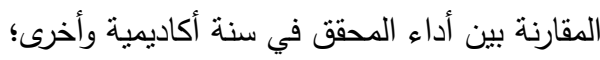

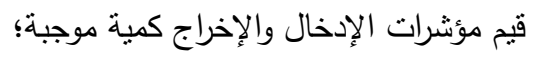
معامل الارتباط قوي موجب بين مؤشرات الإدخال والإخراج المختارة مما يدل على تجانسها ووجود علاقة طردية بينها؛ حجم العينة (عدد وحدات اتخاذ القرار) أكبر من حاصل جَدَاء المدخلات والمخرجات؛ حجم العينة (عدد وحدات اتخاذ القرار) أكبر من ثلاث أضعاف المدخلات والمخرجات؛ مجموع المخرجات والدذخلات أقل من تُلث عدد وحدات

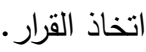

SPSS Statistics 22 المصدر : نتائج برنامج الـ

اتضح من خلال الجدول رقم (Yr) أنّ كافة معاملات الارتباط

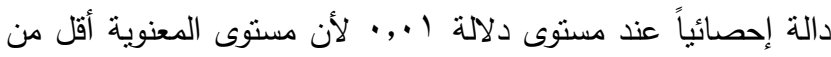

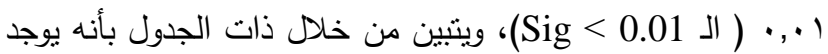
ارتباط موجب قوي يفوق 0.900 (•9 \%) بين جميع متغيرات الإدخال والإخراج، وهذا ما يشير إلى وجود علاقة طردية قوية بين متغيري الإخراج ومتغيرات الإدخال الثلاثة أي أن الزيادة في أحد الإدالي المدخلات أو كلها ستؤدي حتما إلى الزيادة في كمية المخرجين. إضافة إلى وجود علاقة ارتباط قوية موجبة تفوق الـ 0.900 (أي •9 \%)، عند مستوى دلالة ا.,. أيضا بين متغيرات الإدخال الثلاثة فيما بينهما، ومتغيري الإخراج فيما بينهما.

\section{צ. Y. التأكد من تحقق قواعد القوة التقديرية لأسلوب الـ DEA:}

وهي خطوة أساسية ولا تقل أهمية عن الخطوة السابقة، فقبل

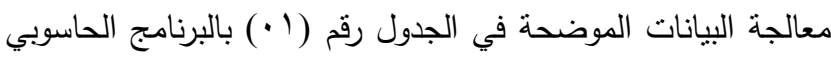
(DEAP Version 2.1) يجب التأكد من مدى تحقق القواعد الأولية

للقوة التقيرية لأسلوب الـ DEA. وفقا للقاعدة الأولى يجب أن يكون:

$$
\begin{aligned}
& {\left[\mathrm{S}_{\mathrm{s}} \geq \mathrm{I}^{*} \mathrm{O}\right] \longrightarrow[16>3 * 2] \longrightarrow[16>6]}
\end{aligned}
$$

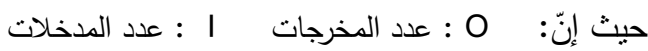

وعليه فإن القاعدة الأولى محققة لأن حاصل جَداء المدخلات والمخرجات أقل من عدد وحدات اتخاذ القرار الداخلة في الدراسة. 
الجزائرية خلال 17 سنة أكاديمية متتالية. تم الحصول على نتائج مؤشر الكفاءة لعوائد الحجم الثابتة (Crste)؛ مؤشر الكفاءة لعوائد الحجم المتغيرة (Vrste)؛ مؤشر الكفاءة الحجمية؛ غلة الحجم E) Scale)

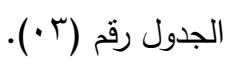

ه. عرض نتائج قياس أداء مؤسسات التعليم العالي الجزائرية

باستخدام أسلوب الـ DEA:

بعد إدراج القيم الكمية لمتغيرات الإدخال والإخراج في البرنامج

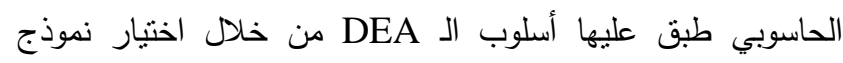
الـBCC بالنوجيه الإدخالي، ومن ثم وقع الاختيار على نموذج الـ

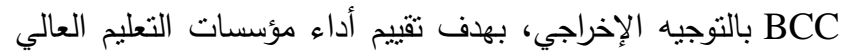

الجدول رقم (ب .) : نتائج قياس أداء مؤسسات التعليم العالي خلال 11 سنة أكاديمية وفقا لنموذجي الـ I-BCC والـ BCC-O.

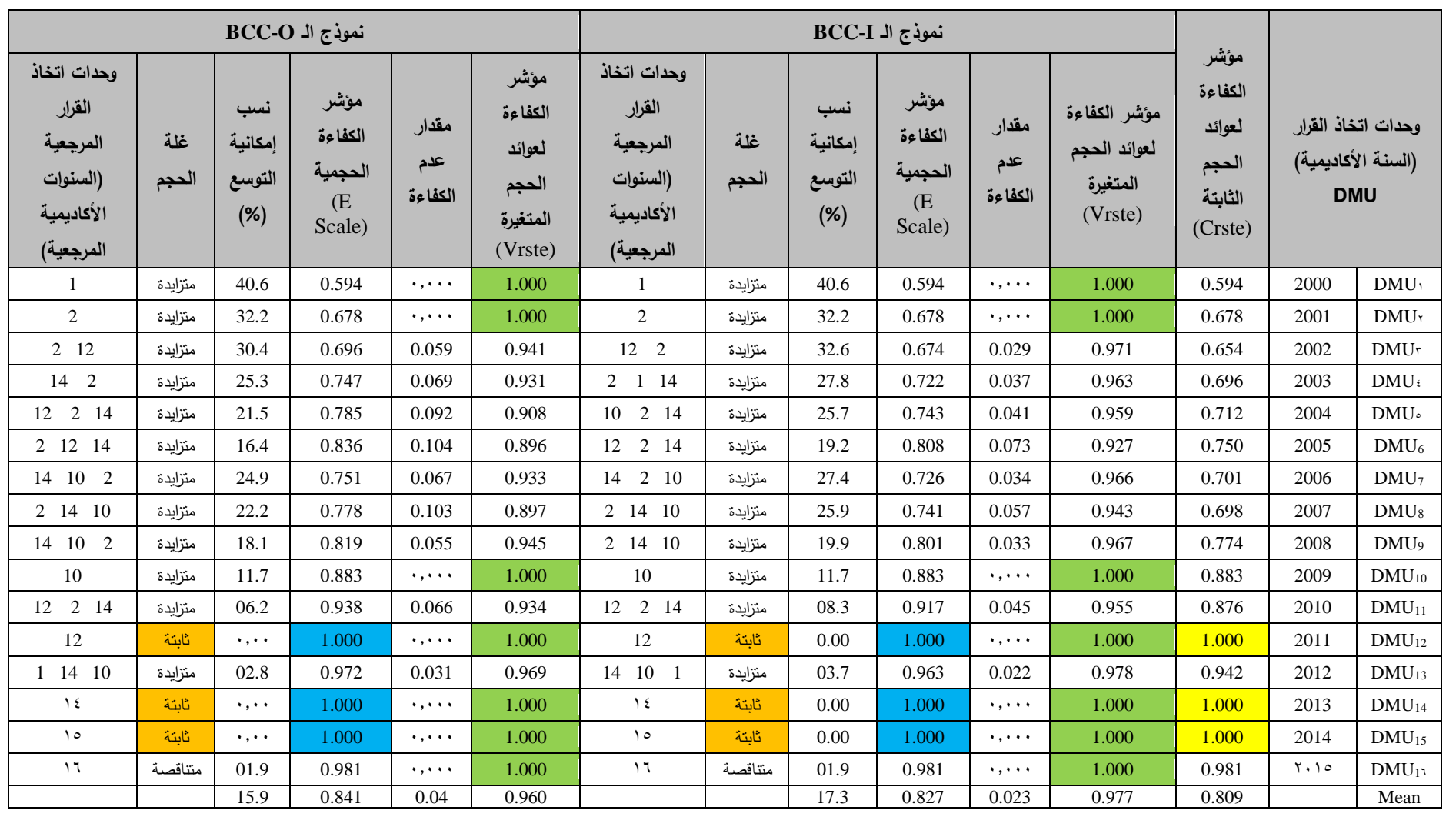

المصدر : نتائج برنامج DEAP Version 2.1 (

צ. مناقثة نتائج قياس أداء مؤسسات التعليم العالي وفقاً

\section{لنموذجي الـ BCC-I و BCC-O}

طبث في البداية نموذج الـ BCC-I والذي يأخذ في الاعتبار التغير في عوائد الحجم من ناحية استخدام أقل كمبة من المدخلات لتحقيق كمية محددة من المخرجات. ومن ثم طبق نموذج ال BCC-O الذي يفترض التغير في عوائد الحجم، من ناحية تعظيم المخرجات باستخدام المدخلات المتاحة فعلا. يعطي نموذج الـ BCC بكلا التوجيهين القيمة واحد ( . . , أو

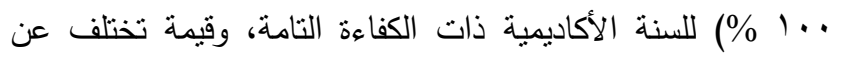
الواحد للسنة الأكاديمية غير الكفؤة. ومن خلال مختلف مؤشرات الكفاءة النسبية والكفاءة الحجمية الموضحة في الجدول رقم (ب •) تم

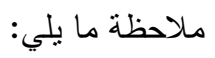
يوجد تباين في معدلات كفاءة (أداء) مؤسسات التعليم العالي الجزائرية بين سنة أكاديمية وأخرى بالتوجيه الإدخالي
قبل منافثة نتائج تقييم الأداء وفقا لنموذجي الـ BCC-I و الـ BCC-O أولاً من مدى تحقق القاعدة الثالثة للقوة التقديرية لأسلوب الـ DEA.

$$
\text { القاعدة الثالثة : م م }
$$

Eff DMUs $\leq 1 / 3 * \mathrm{~S}_{\mathrm{s}} \rightarrow 3 \leq 1 / 3 * 16 \rightarrow 3<5.33$

القاعدة الثالثة محققة، مما يعني أنّ حجم العينة مقبول لأن عدد وحدات اتخاذ القرار أو عدد السنوات الأكاديمية ذات الكفاءة التامة وفقا لمؤشر الـ Vrste أقلّ تماماً من ثلث عدد السنوات الأكاديمية الداخلة في الدراسة. وبما أن كافة شروط وقواعد القوة التقديربة محققة فهذا بجعل نتائج تقييم الأداء المتحصل عليها باستخدام أسلوب الـ DEA دقيقة وصادقة. هذه النتائج تم تحليلها وتفسيرها ومقارنتها كما يلي: 


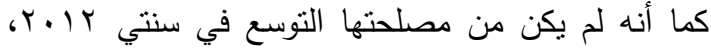
و 10 ـ ب وكان عليها المحافظة على مستوى أدائها الأمتل. مؤسسات التعليم العالي الجزائرية تعمل عند غلة حجم

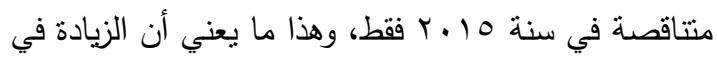
مخرجاتها في هذه السنة نطلبت منها استخدام كميات أكبر

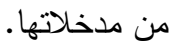

وفقا لنموذج الـ BCC-I فإن سنة ....

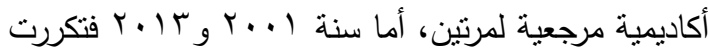
كسنة مرجعية لثماني سنوات أكاديمية غير كفؤة، بينما سنة

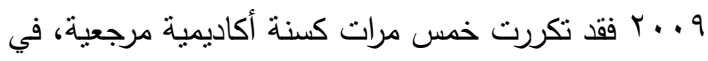
حين أن سنة 11 ـ ب قد تكررت ثلات مرات فقط. وفقا لنموذج الـ BCC-O سنة .... نكررت كسنة

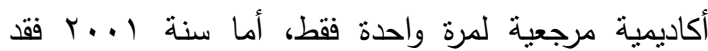

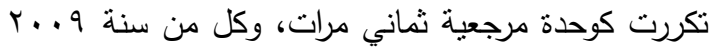

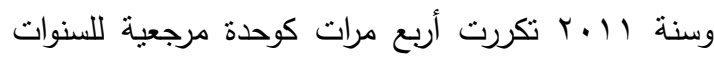

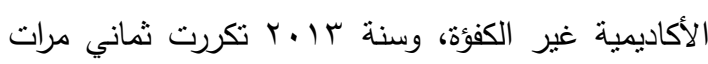
كسنة مرجعية للسنوات الأكاديمية غير الكفؤة.

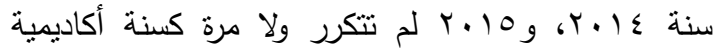
مرجعية لباقي السنوات الأكاديمية غير الكفؤة وفقا اللنموذجين.

هذه الملاحظات التي نم الاثشارة إليها أعلاه، والتي تم التوصل إليها من خلال قراءة نتائج الجدول رقم (r.)، يمكن تفسيرها من خلال الجدول رقم (ع.) والذي وضحت فيه كميات الدذخلات الفائضة والمخرجات الراكدة وفقا لكل سواء من ناحية ندنية ددخلاتها

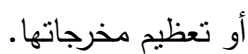

كمبات المدخلات الفائضة، والمخرجات الراكدة في السنوات

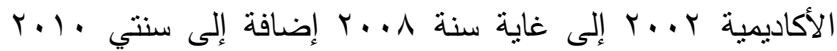

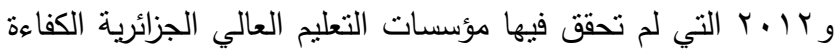
التامة، وفقا لهلف تدنية المدخلات وهدف تعظيم المخرجات كما هو

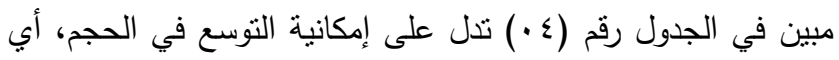
إمكانية تحقيق مخرجات بكميات أكبر من كميات المخرجات المحققة فعليا والموضحة في الجدول رقم (r.) باستخدام كميات أقل من

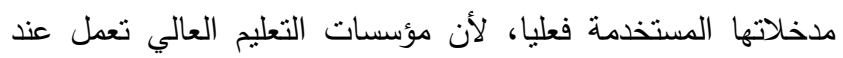

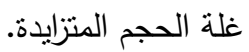
كما أن الكميات الفائضة من المدخلين الأول والثاني المتمثلين في إجمالي الطلبة المسجلين في مرحلة التدرج، وإجمالي الطلبة

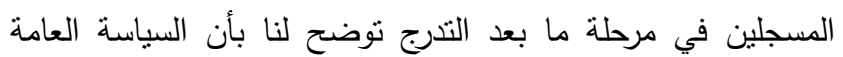

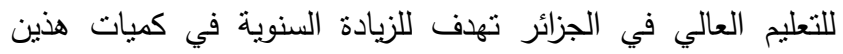

مؤسسات التعليم العالي الجزائرية حققت الكفاءة التامة في كي سبع سنوات أكاديمية وفقا لمؤنشر الـ Vrste في كلا

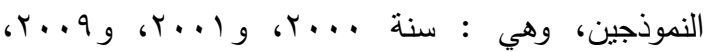
و

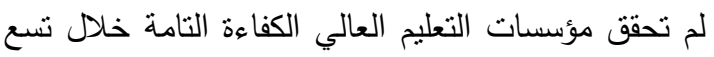
سنوات أكاديمية لا بمؤشر عوائد الحجم الثابتة ولا المتغيرة

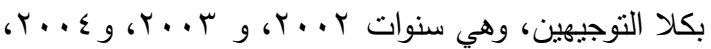

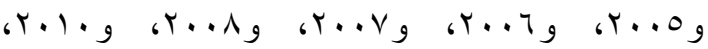
.$r \cdot r^{2}$

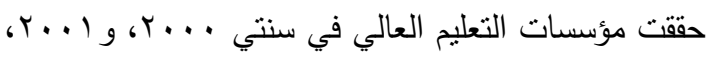

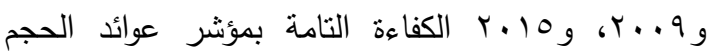
المتغيرة، ولم تحقق الكفاءة بمؤشر الـ Crste مما يؤكد لنا

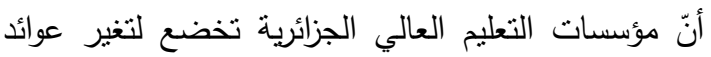
الحجم بين سنة أكاديمية وأخرى. مؤشرات الـ Vrste للسنوات الأكاديمية غير الكفؤة كانت متباينة في كلا النموذجين، وقريبة نسيباً من معدل الكفاءة التام (أي قريبة من ., (1).

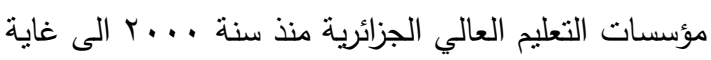

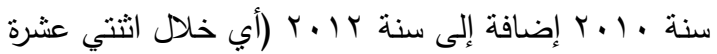
سنة أكاديمية) كانت تعمل عند عوائد الحجم المتزايدة

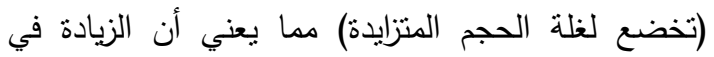

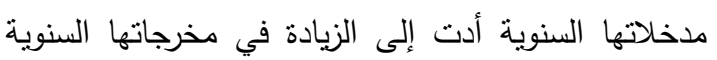
بنسبة أكبر من النسبة التي زادت بها المدخلات. وعليه ففي هذه السنوات كان بإمكان مؤسسة التعليم العالي الجزائرية التوسع في حجم انتاجها، هذا التوسع يكون بنسب متفاوتة بين سنة أكاديمية غير كفؤة، وأخرى كما هو موضح لتحنح في العمود السابع والعمود الثالث عشر من الجدول رقم

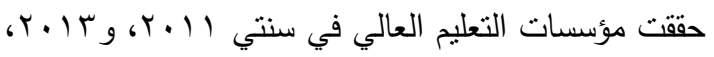
و ع ا.بr الكفاءة التامة حسب مؤشر عوائد الحجم الثابتة، ومؤشر عوائد الحجم المتغيرة، وحتى معدل الكفاءة الحجمية

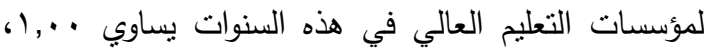

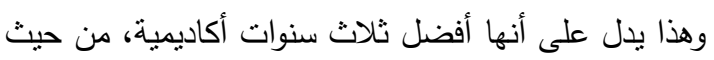
كفاءة العطليات الداخلية، والكفاءة الكلية لمؤسسات التعليم العالي الجزائرية، وأن مؤسسات التعليم العالي في هذه السنوات استخدمت كافة مدخلاتها تحقق مخرجاتها الفعلية، 
أما بالنسبة لباقي السنوات الأكاديمية التي لم تحقق فيها مؤسسات التعليم العالي الكفاءة التامة وكان بمقدورها استخدام كميات أقل من مدخلاتها لتحقيق نفس مخرجاتها المحددة أو حتى تعظيمها، فقد اتضح من خلا نتائج المدخلات الفائضة، والمخرجات الراكدة، والقيم المستهدفة الموضحة في الملحق رقم (1 ( ) بأنه في الفترة

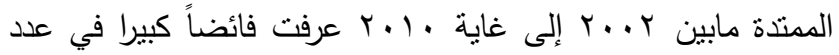

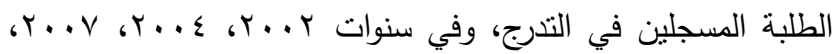

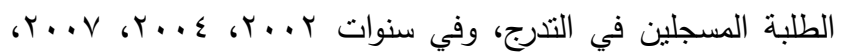

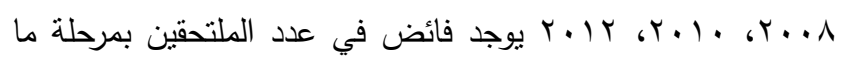
بعد التدرج، أما المدخل الثالث المتمنل في الأساتذة الدائمين فسجلت فيه فوائض في سنة س . . r فقط وفي باقي السنوات فقد استخدمت كافة كمياته لتحقيق المخرجات الفعلية وكان بالإمكان استخدام نفس ولى كمياته لتعظيم كمية المخرجات أيضا.
المدخلين، مع اغفال الحاجة لتعظيم المخرجات وخاصة المخرج الثاني المتمثل في إجمالي المنشورات العلمية. إذا ومن خلال استعراض نتائج تقييم الأداء الكمي لمؤسسات التعليم العالي كوحدة واحدة وفقا لنموذج عوائد الحجم المتغيرة بالتوجيهين الإدخالي والإخراجي، يمكن القول بأن أداء مؤسسات

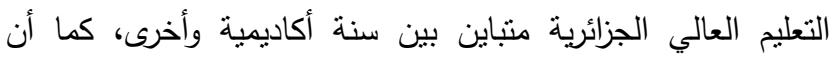
مؤسسات التعليم العالي الجزائرية استطاعت استخدام مدخلاتها الفعلية

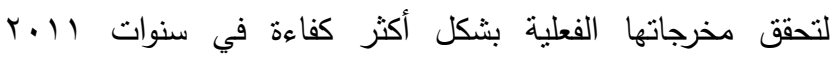

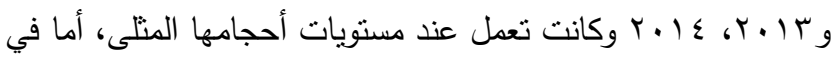
سنوات . . . r و 1 . . r و 9 . . . ب بالرغم من تحقيقها لمخرجاتها الفعلية باستخدام مدخلاتها الفعلية إلا أنه كان بمقدورها التوسع في حجم مخرجاتها أي كان من الممكن تحقيق مخرجات أكثر من خلا استخدام كمية مدخلاتها الفعلية.

الجدول رقم (ع · ) : كميات المدخلات الفائضة والمخرجات الراكدة وفقا لنموذجي الـ BCC-I و الـ BCC-O.

\begin{tabular}{|c|c|c|c|c|c|c|c|c|c|c|c|}
\hline \multicolumn{5}{|c|}{ نموذج الـ BCC-O } & \multicolumn{5}{|c|}{ نموذج الـ BCC-I } & \multirow{3}{*}{\multicolumn{2}{|c|}{ DMU }} \\
\hline \multicolumn{2}{|c|}{ المخرجات الراكدة } & \multicolumn{3}{|c|}{ المدخلات الفائضة } & \multicolumn{2}{|c|}{ المخرجات الراكدة } & \multicolumn{3}{|c|}{ المدخلات الفائضة } & & \\
\hline Output $_{2}$ & Output $_{1}$ & Input $_{3}$ & Input $_{2}$ & Input $_{1}$ & Output $_{2}$ & Output $_{1}$ & Input $_{3}$ & Input $_{2}$ & Input $_{1}$ & & \\
\hline$\cdot, \cdots$ & $\cdot, \cdots$ & $\cdot, \cdots$ & $\cdot, \cdots$ & $\cdot, \cdots$ & $\cdot, \cdots$ & $\cdot, \cdots$ & $\cdot, \cdots$ & $\cdot, \cdots$ & $\cdot, \cdots$ & 2000 & DMU, \\
\hline$\cdot, \cdots$ & $\cdot, \cdots$ & $\cdot, \cdots$ & $\cdot, \cdots$ & $\cdot, \cdots$ & $\cdot, \cdots$ & $\cdot, \cdots$ & $\cdot, \cdots$ & $\cdot, \cdots$ & $\cdot, \cdots$ & 2001 & $\mathrm{DMU}_{r}$ \\
\hline $11 \cdot, r$ & $\cdot, \cdots$ & $\cdot, \cdots$ & $9 \wedge \cdot, 7$ & rฯAV०, & $V O, Y$ & $\cdot, \cdots$ & $\cdot, \cdots$ & $11 \vee q, r$ & rчrqт,0 & 2002 & $\mathrm{DMU}_{r}$ \\
\hline 0,17 & $\cdot, \cdots$ & 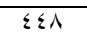 & $\cdot, \cdots$ & TAYVY,O & $\cdot, \cdots$ & $\cdot, \cdots$ & $1 \Gamma 4,1$ & $\cdot, \cdots$ & 79999,1 & 2003 & $\mathrm{DMU}_{\varepsilon}$ \\
\hline$\cdot, \cdots$ & $\cdot, \cdots$ & $\cdot, \cdots$ & rরY,q & $\varepsilon \wedge 977, \Gamma$ & $\cdot, \cdots$ & $\cdot, \cdots$ & $\cdot, \cdots$ & $1 \wedge r, 7$ & rro79,r & 2004 & DMU。 \\
\hline $19 V, V$ & $\cdot, \cdots$ & $\cdot, \cdots$ & $\cdot, \cdots$ & 9Y7Tะ,0 & $\vee \wedge, r$ & $\cdot, \cdots$ & $\cdot, \cdots$ & $\cdot, \cdots$ & VY970, & 2005 & $\mathrm{DMU}_{6}$ \\
\hline$\cdot, \cdots$ & $\varepsilon \leqslant \wedge \cdot, 7$ & $\cdot, \cdots$ & $\cdot, \cdots$ & TYMr., & $\cdot, \cdots$ & $00.1, \pi$ & $\cdot, \cdots$ & $\cdot, \cdots$ & YEVTr, & 2006 & $\mathrm{DMU}_{7}$ \\
\hline$\cdot, \cdots$ & $\cdot, \cdots$ & $\cdot, \cdots$ & IATV,A & $\sum \| \wedge \wedge, 1$ & $\cdot, \cdots$ &,,$\cdots$ & $\cdot, \cdots$ & $I \leqslant Y \wedge, Y$ & $19 r V 7,1$ & 2007 & $\mathrm{DMU}_{8}$ \\
\hline$\cdot, \cdots$ & $\cdot, \cdots$ & $\cdot, \cdots$ & rTוq,r & $11 \vee 910, r$ & $\cdot, \cdots$ & $\cdot, \cdots$ & $\cdot, \cdots$ & $\Gamma \cdot \leq \wedge, 1$ & I.rYIA & 2008 & $\mathrm{DMU}_{9}$ \\
\hline$\cdot, \cdots$ & $\cdot, \cdots$ & $\cdot, \cdots$ & $\cdot, \cdots$ & $\cdot, \cdots$ & $\cdot, \cdots$ & $\cdot, \cdots$ & $\cdot, \cdots$ & $\cdot, \cdots$ & $\cdot, \cdots$ & 2009 & $\mathrm{DMU}_{10}$ \\
\hline$\cdot, \cdots$ & $\cdot, \cdots$ & $\cdot, \cdots$ & TIrV,A & $110 \times 10,1$ & $\cdot, \cdots$ & $\cdot, \cdots$ & $\cdot, \cdots$ & $7 \leq 77,7$ & $11 \vee 9 \wedge 1, v$ & 2010 & $\mathrm{DMU}_{11}$ \\
\hline$\cdot, \cdots$ & $\cdot, \cdots$ & $\cdot, \cdots$ & $\cdot, \cdots$ & $\cdot, \cdots$ & $\cdot, \cdots$ &,,$\cdots$ & $\cdot, \cdots$ & $\cdot, \cdots$ & $\cdot, \cdots$ & 2011 & $\mathrm{DMU}_{12}$ \\
\hline$\cdot, \cdots$ & $9 \leq 7 \leq, \Gamma$ & $\cdot, \cdots$ & ovo & $\cdot, \cdots$ & $\cdot, \cdots$ & $9 \wedge \vee 9,1$ & $\cdot, \cdots$ & $7 \wedge 7, Y$ & $\cdot, \cdots$ & 2012 & $\mathrm{DMU}_{13}$ \\
\hline$\cdot, \cdots$ & $\cdot, \cdots$ & $\cdot, \cdots$ & $\cdot, \cdots$ & $\cdot, \cdots$ & $\cdot, \cdots$ & $\cdot, \cdots$ & $\cdot, \cdots$ & $\cdot, \cdots$ & $\cdot, \cdots$ & 2013 & $\mathrm{DMU}_{14}$ \\
\hline$\cdot, \cdots$ & $\cdot, \cdots$ & $\cdot, \cdots$ & $\cdot, \cdots$ & $\cdot, \cdots$ & $\cdot, \cdots$ & $\cdot, \cdots$ & $\cdot, \cdots$ & $\cdot, \cdots$ & $\cdot, \cdots$ & 2014 & $\mathrm{DMU}_{15}$ \\
\hline$\cdot, \cdots$ & $\cdot, \cdots$ & $\cdot, \cdots$ & $\cdot, \cdots$ & $\cdot, \cdots$ & $\cdot, \cdots$ & $\cdot, \cdots$ & $\cdot, \cdots$ & $\cdot, \cdots$ & $\cdot, \cdots$ & $r .10$ & $\mathrm{DMU}_{17}$ \\
\hline
\end{tabular}

المصدر : نتائج برنامج DEAP Version 2.1 .

خاتمة:

\section{المراجع:}

1. The Institute of Chartered Accountants of Scotland (ICAS). What is performance? Published by the Technical Policy Board of ICAS. May 2016; P 1-20. Last site visit 18/06/2019. Available at https://www.icas.com/_data/assets/pdf_file/ 0004/243967/ICAS-What-isperformance.pdf.

2. Farrell M.J. The Measurement of Productive Efficiency. Journal of the Royal Statistical Society. 1957; Series A (Part III, Vol 120, $\mathrm{N}^{\circ}$ 3) : $\mathrm{P}$ 253-290 . Last site visit 10/04/2016. Available at : https://links.jstor.org/sici?sici=00359238\%281957\%29120\%3A3\%3C253\%3AT MOPE\%3E2.0.CO\%3B2-U.
من خلال هذه الدراسة تم توضيح مدى فعالية الاعتماد على أسلوب التحليل التطويقي للبيانات في تقييم أداء مؤسسات التعليم العالي الجزائرية، ورغم استخدام لخمس مؤشرات إدخال وإخراج بقيم كمية ومحدودة نوعا ما وتعكس بعدي التدريس والبحث العلمي فقط، ولا تعكس بعد خدمة المجتمع، وجودة البحث العلمي، إلاّ أن النتائج التي توصلت إليها الدراسة تبقى مفيدة لمختلف أصحاب المصلحة وصانعي السياسات العامة في قطاع التعليم العالي الجزائري خاصة، وفي باقي مؤسسات التعليم العالي بالوطن العربي، لأن ما تم التوصل إليه من نتائج يسهل عليهم عملية توزيع وتخصيص الموارد مستقبلا، كما يوفر لهم طريقة متلى للتقييم والمقارنة بين أداء الجامعات، والمعاهد، والكليات، والأقسام والوقوف على أسباب عدم كفاءة كل منها

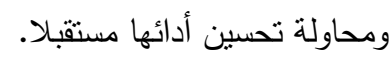


3. Kaftroodya Hosein Rad \& Aminnaserib Fatemeh. Data Envelopment Analysis (DEA): Case Study of the Iranian Universities. Indian Journal of Fundamental and Applied Life Sciences. 2014; Vol 4 (S1): P 1045-1050. Last site visit 20/06/2019. Available at :http://www.cibtech.org/sp.ed/j1s/2014/01/00 (123).pdf.

4. Cooper W. William \& Seiford M. Lawrence \& Zhu Joe. Handbook on Data Envelopment Analysis, International Series in Operations Research \& Management Science. 2011; Second Edition (Springer Science+Business Media, Vol 164 : P 1-524. Last site visit 20/06/2019. Available at : http://www.springer.com/series/6161.

5. Abbott Malcolm \& Doucouliagos Chris. The efficiency of Australian universities: a data envelopment analysis. Economics of Education Review. February 2003; $\mathrm{N}^{\circ} 22$ (1): P 89-97. Last site visit 20/06/2019. Available at: https://ideas.repec.org/a/eee/ecoedu/v22y20 03i1p89-97.html.

6. Rosenmayer Tomáš. Using Data Envelopment Analysis : a Case of Universities. Review of Economic Perspectives - NÁRODOHOSPODÁŘSKÝ OBZOR. March 2014; Vol 14 (ISSUE 1) : P 34-54. Last site visit 18/06/2019. Available at

https://www.researchgate.net/publication/27 5998662_Using_Data_Envelopment_Analys is_A_Case_of_Universities.

7. Hayder Abbas Drebee \& Nasir Ibrahim Nasser. Using Data Envelopment Analysis (DEA) to Measure the Relative Efficiency of Secondary Schools at Al-Qadisiyah Province-Iraq. MJAES. 2015; 7 (3): P 260280. Last site visit 12/06/2019. Available at: https://www.iasj.net/iasj?func=fulltext\&aId=134 553.

8. Charnes. A \& Cooper. W. William \& Rhodes Edwardo. Measuring the efficiency of decision making units. North-Holland Publishing Company. European Journal of Operational Research. 1978; (2): P 429-444. Last site visit 18/06/2019. Available at : https://www.utdallas.edu/ ryoung/phdsemin ar/CCR1978.pdf.

9. Marti. M. Milan \& Novakovic. S. Marina \& Baggia Alenka. Data Envelopment Analysis - Basic Models and their Utilization. Organizacija. March-April 2009; Vol $42\left(\mathrm{~N}^{\circ}\right.$ 2) : P 37-43. Last site visit 17/06/2019. Available http://faratarjome.ir/u/media/shopping_files/ store-EN-1459861462-4250.pdf.

10. Moudher Khaled Abdelhzmid. Using of Data Envelopment Analysis (DEA) in Measuring the Efficiency of the Education Vocational Schools (Industrial Branch as a Model): Exploratory Study. The Tikrit Journal of Administrative Sciences. 2017; 4(40): P 102-128. Last site visit 12/06/2019. Available at: https://www.iasj.net/iasj?func=fulltext $\&$ aId $=140$ 117.

11. Manzoni Alex. A New Approach To Performance Measurement Using Data Envelopment Analysis: Implications for Organisation Behaviour, Corporate Governance and Supply Chain Management. Thesis submitted in partial fulfillment for the degree of Doctor of Business Administration. Faculty of Business and Law. Victoria University. March 2007; P 1289. Last site visit 16/06/2019. Available at : https://core.ac.uk/download/pdf/10827151.p df.

12. Montoneri Bernard. Teaching Improvement Model Designed with DEA Method and Management Matrix. The IAFOR Journal of Education. Winter 2014; Vol 2 (Issue 1): P 125-155. Last site visit 13/06/2019. Available at :

https://files.eric.ed.gov/fulltext/EJ1080351.p df.

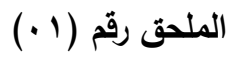

قيم المدخلات وإلمخرجات المستهدفة وفقا لنموذج الـCCR

Input orientated DEA

scale assumption: VRS

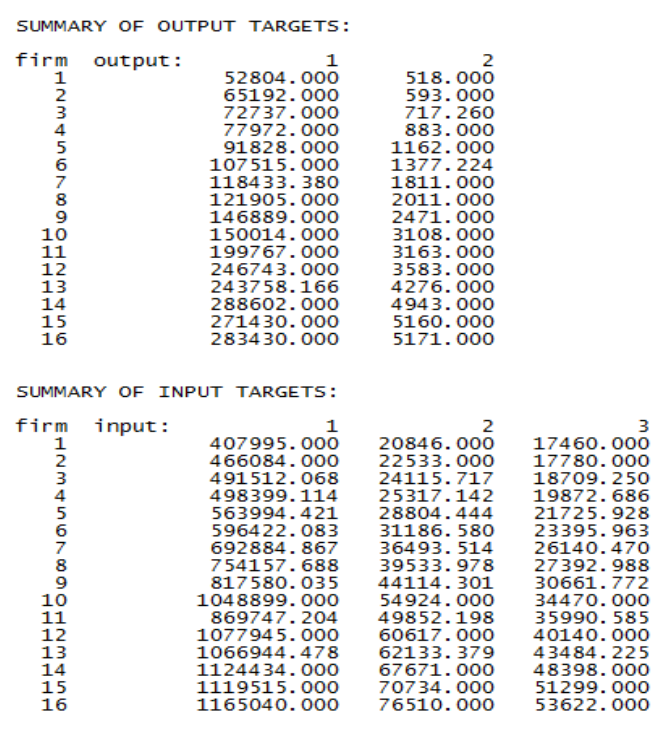

المصدر : نتائج برنامج 2.1 DEAP Version 
قيم المدخلات والمخرجات المستهدفة وفقا لنموذج الـBCC

\section{output orientated DEA}

\section{scale assumption: VRS}

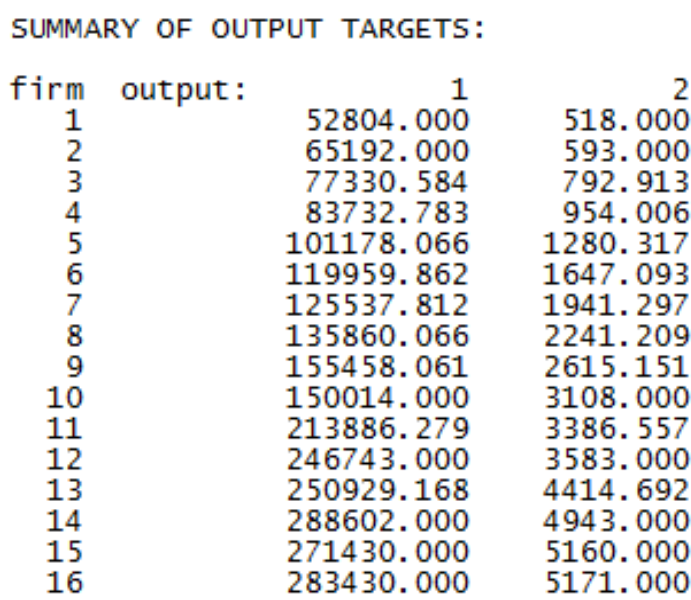

SUMMARY OF INPUT TARGETS:

$\begin{array}{rrrr}\text { firm } & \text { input: } & 1 & 2 \\ 1 & 407995.000 & 20846.000 & 17460.000 \\ 2 & 466084.000 & 22533.000 & 17780.000 \\ 3 & 506993.311 & 25079.314 & 19275.000 \\ 4 & 520720.428 & 26279.000 & 20320.986 \\ 5 & 574013.681 & 29838.079 & 22650.000 \\ 6 & 629208.414 & 33630.000 & 25229.000 \\ 7 & 710723.830 & 37787.000 & 27067.000 \\ 8 & 779278.876 & 41620.177 & 29062.000 \\ 9 & 834081.784 & 45444.640 & 31703.000 \\ 10 & 1048899.000 & 54924.000 & 34470.000 \\ 11 & 918597.861 & 52837.116 & 37688.000 \\ 12 & 1077945.000 & 60617.000 & 40140.000 \\ 13 & 1090592.000 & 63636.905 & 44448.000 \\ 14 & 1124434.000 & 67671.000 & 48398.000 \\ 15 & 1119515.000 & 70734.000 & 51299.000 \\ 16 & 1165040.000 & 76510.000 & 53622.000\end{array}$

